\title{
Isolation of “Treponema Hyodysenteriae" strains and control of their pathogenicity on healthy piglets. Comparison with results given by dysentery obtained from spontaneous contamination in pens
}

\author{
J. P. RAYNAUD, G. BRUNAULT, J. PHILIPPE \\ Station de Recherche et de Développement Vétérinaire et Nutrition Animale, \\ Pfizer International, 37400 Amboise (France)
}

Forty two young weaned piglets weighing $\mathrm{I} 2 \mathrm{~kg}$ on an average were orally inoculated with 4 strains of Treponema isolated from clinical cases of dysentery and producing a complete or a beta haemolysis as described for T. Hyodysenteriae (pathogenic strains). Despite this, one of the four strains was found to be non pathogenic. This was also observed in animals during treatment when velapses of dysentery occurred. The main characteristics of dysentery produced by the pathogenic strains of $T$. hyodysenteriae are the following: high morbidity ( $76 \mathrm{p}$. I 00 of the inoculated animals) and very high mortality (93 p. Ioo of the animals; 7 p. I0o with spontaneous cure). For those dead from dysentery, the death occurred on day 20 after inoculation, after 8 days of dysentery and 2 days of diarrhoea with mucus and without blood. The disease transmitted by $T$. hyodysenteriae is equivalent to the one obtained by spontaneous contamination in pens both as regards the samples taken, the incubation time and the length of each typical phase of the dysentery.

\section{Swine dysentery: development of a severe S.D. model combining oral infection and continuous in pen-contamination (O.I. + G.P.C.) for the assay of drugs preventing the disease}

\author{
J. P. RAYNAUd (1), G. BRUNAULT( $(1)$, E. B. P. PATTERSON( $\left.{ }^{2}\right)$ \\ (1) Station de Recherche et de Développement Vétérinaire et Nutrition Animale, \\ Pfizer International, 37400 Amboise. (France) \\ (2) A gricultural Development Division, Pfizer International, \\ New-York Toor7 (U.S.A.)
}

\begin{abstract}
A new experimental Swine dysentery (S.D.) model combining oral infection and continuous in pen-contamination (O.I. + C.P.C.) used to determine the efficiency of drugs gave results similar to those obtained in the fields. The efficiency of preventing a severe manifestation of the disease, the possible effect of stress on apparently well protected animals and the possible recurrence of the disease after drug withdrawal were investigated. Each experimental protocol required 82 to 106 pigs and 4 protocols were applied. The efficiency of the best three drugs were: carbadox $50 \mathrm{ppm}+$ Sulfamethazine $100 \mathrm{ppm}>$ carbadox $50 \mathrm{ppm}>$ olaquindox $100 \mathrm{ppm}$. Others tested in the same conditions were less efficient: ASP $250 \mathrm{ppm}\left({ }^{*}\right)$, ronidazole $60 \mathrm{ppm}$ or lincomycin + spectinomycin $44 \mathrm{ppm}$.
\end{abstract}
I.N.R.A.
BIBLIOTHEQUE UO 35906 DOMAINE DE CROUELLE
(*) ASP (chlortetracycline + sulfamethazine + penicilline). 